Sebastian Fuchs

Unternehmensreputation und Markenstärke 
GABLER EDITION WISSENSCHAFT 
Sebastian Fuchs

\section{Unternehmensreputation und Markenstärke}

Analyse von Wechselwirkungen und Ansätzen zur Prognose des Konsumentenverhaltens

Mit einem Geleitwort von Prof. Dr. Manfred Schwaiger 
Bibliografische Information der Deutschen Nationalbibliothek

Die Deutsche Nationalbibliothek verzeichnet diese Publikation in der

Deutschen Nationalbibliografie; detaillierte bibliografische Daten sind im Internet über $<$ http://dnb.d-nb.de> abrufbar.

Dissertation Ludwig-Maximilians-Universität München, 2009

D 19

1. Auflage 2009

Alle Rechte vorbehalten

(C) Gabler I GWV Fachverlage GmbH, Wiesbaden 2009

Lektorat: Frauke Schindler / Britta Göhrisch-Radmacher

Gabler ist Teil der Fachverlagsgruppe Springer Science+Business Media.

www.gabler.de

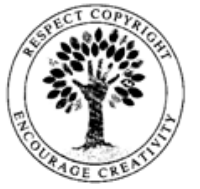

Das Werk einschließlich aller seiner Teile ist urheberrechtlich geschützt. Jede Verwertung außerhalb der engen Grenzen des Urheberrechtsgesetzes ist ohne Zustimmung des Verlags unzulässig und strafbar. Das gilt insbesondere für Vervielfältigungen, Übersetzungen, Mikroverfilmungen und die Einspeicherung und Verarbeitung in elektronischen Systemen.

Die Wiedergabe von Gebrauchsnamen, Handelsnamen, Warenbezeichnungen usw. in diesem Werk berechtigt auch ohne besondere Kennzeichnung nicht zu der Annahme, dass solche Namen im Sinne der Warenzeichen- und Markenschutz-Gesetzgebung als frei zu betrachten wären und daher von jedermann benutzt werden dürften.

Umschlaggestaltung: Regine Zimmer, Dipl.-Designerin, Frankfurt/Main

Gedruckt auf säurefreiem und chlorfrei gebleichtem Papier

Printed in Germany

ISBN 978-3-8349-1504-7 


\section{Geleitwort}

Selbst in Zeiten der Finanzkrise wird der Marktwert eines im HDAX gelisteten Unternehmens durchschnittlich immerhin noch zu mehr als einem Drittel von immateriellen Werten bestimmt, im Mittel der Jahre 2000 bis 2007 lag dieser Anteil sogar bei mehr als zwei Drittel. Zu den immateriellen Vermögensgegenständen eines Unternehmens gehören auch die Unternehmensreputation und die Stärke seiner Marken. Zwischen der Reputation eines Unternehmens und der Stärke seiner Marken werden Zusammenhänge vermutet, die Herr Fuchs in der vorliegenden Dissertation überprüft. Er bewegt sich damit auf wissenschaftlich weitgehend unerforschtem Terrain; zwar existieren vor allem in der amerikanischen Literatur Beiträge, die die Unternehmensreputation als „umbrella-brand“ beschreiben, der die Einführung neuer Marken aus Sicht eines Unternehmens einfacher gestaltet, eine exakte Analyse der Wechselbeziehungen zwischen Unternehmensreputation und Markenstärke ist jedoch bisher nicht erfolgt.

Die hier angestellte Untersuchung wird motiviert durch die Annahme, dass gängige Markenmanagementmodelle Verhaltensaspekte des dahinter stehenden Unternehmens (wie z.B. die gerade in jüngerer Zeit intensiv diskutierten Corporate-Social-Responsibility-Aktivitäten) nicht einbeziehen. Es stellt sich somit die Frage, ob in einem Modell, das neben gängigen MarkenstärkeKonstrukten auch Verhaltenskomponenten des Unternehmens abbildet, ein höherer Anteil der Varianz von so genannten „product-market outcomes“ - bestehend aus Kaufintention, Mehrpreisbereitschaft und Präferenz einer Marke gegenüber Konkurrenzprodukten - erklärt werden kann. Eine derartige Untersuchung muss nach Markenarchitektur differenziert erfolgen, denn bei Produktmarken (die im Extremfall von einem „House of Brands“ wie z.B. Procter\&Gamble geführt werden) ist nicht zwangsweise bekannt, welches Unternehmen hinter dieser Marke steht, so dass nicht angenommen werden darf, dass das Verhalten des Unternehmens in gleichem Ausmaß auf die Wahrnehmung der Markenstärke respektive der Unternehmensreputation Einfluss hat wie bei Unternehmensmarken (z.B. IBM). 
Aus methodischer Sicht finden sich in der vorliegenden Arbeit anwendungsorientierte Einblicke in die Modellierung latenter Konstrukte in Strukturgleichungsmodellen - und hier insbesondere in die Behandlung unbeobachtbarer Heterogenität mit Hilfe des FIMIX-PLS-Ansatzes - sowie der impliziten Messung von Präferenzen mittels Conjoint-Analysen mit Fokus auf Choice-based Conjoint-Analysen unter Einsatz des Hierarchischen Bayes-Algorithmus. Zur Hypothesenprüfung wird des Weiteren ein anspruchsvolles Experimentaldesign eingesetzt.

Die Ergebnisse aus einer umfangreichen deutschlandweiten Onlinebefragung geben Antwort auf die Frage, ob ein Reputationsmodell oder ein Markenstärkemodell bei Unternehmensmarken einen höheren Varianzanteil der productmarket outcomes (PMO) erklärt. Es stellt sich heraus, dass das Markenstärkemodell in der Lage ist, einen höheren Anteil der Varianz der PMO (und somit das Konsumentenverhalten) zu erklären als das Reputationsmodell. Die Anreicherung des Markenstärkemodells um Reputationsdimensionen (Sympathie und Kompetenz) führt jedoch zu einer signifikanten Verbesserung der Prognosekraft.

Mithilfe eines geeigneten Experimentaldesigns weist Herr Fuchs für Produktmarken nach, dass eine Veränderung der Reputation eines Unternehmens eine gleichgerichtete Veränderung der Stärke seiner Produktmarken bewirkt und vice versa. Abgelehnt werden dagegen Hypothesen, denen zufolge diese Veränderungsstärken abhängig sind vom Vorwissen des Konsumenten.

Die vorliegende Arbeit kann nicht nur eine wissenschaftliche Lücke schließen, sie kann auch den praktischen Dialog bereichern und dazu beitragen, sowohl das Marken- als auch das Reputationsmanagement in den Unternehmen weiter zu verbessern.

Prof. Dr. Manfred Schwaiger 


\section{Vorwort}

Reputation und Markenstärke sind sich sehr ähnlich. Beide bezeichnen in wirtschaftswissenschaftlicher Hinsicht jeweils eine Größe, die die Einstellung gegenüber einem Objekt des Wirtschaftsgeschehens umschreibt. Im Bezug auf ihre Definition sind beide zudem vom Betrachter abhängig. Vielfältig sind deshalb auch die Ansätze und Institutionen (Agenturen, Beratungsunternehmen, wissenschaftliche Institute), die versuchen den Phänomenen Marke und Reputation eines Unternehmens einen Wert zuzuweisen. Niemand würde die Verwandtschaft der beiden leugnen - umso erstaunlicher ist, dass oben genannte Institutionen ihre Verbindung meist scheuen. Dabei zeigt die Vergangenheit, dass Übernahmen von Marken sowie Fusionen und Umbenennungen von Unternehmen die Einstellungen von Betrachtern verändern. Fraglich ist nun, welche Konsequenzen die Verwandtschaft der beiden Größen haben kann. Für Marken, die für ein ganzes Unternehmen stehen (Unternehmensmarken wie z.B. IBM oder Ford) interessiert, ob denn eine „richtige“ Perspektive existiert: Soll sich ein Unternehmen der Messung und Steuerung seiner Reputation widmen oder doch lieber der seiner Markenstärke? Für Marken, die sich namentlich von ihren anbietenden Unternehmen unterscheiden (Produktmarken wie z.B. Fanta aus dem Hause Coca-Cola), stellt sich hingegen die Frage, ob die Verbindung der Reputation eines Unternehmens mit einer seiner Marken messbare Folgen hat. Falls ja, würde dies bedeuten, dass eine losgelöste Betrachtung von Markenstärke und Unternehmensreputation falsch ist.

Um diese Fragen beantworten zu können, wurden im Rahmen dieser Arbeit nach Abgrenzung und Definition der beiden betrachteten Größen zwei empirische Studien durchgeführt: Eine Onlinebefragung, die den Erklärungsgehalt der beiden Größen Unternehmensreputation und Markenstärke bei Unternehmensmarken direkt vergleicht, sowie ein Experiment, das die Auswirkungen der Veränderung einer der beiden Größen auf die jeweils andere Größe bei Produktmarken quantifizierbar macht.

Die Arbeit wurde während meiner Tätigkeit als wissenschaftlicher Mitarbeiter am Institut für Marktorientierte Unternehmensführung (IMM) der LudwigMaximilians-Universität München angefertigt. Das Gelingen dieses Vorhabens 
habe ich jedoch nicht ausschließlich mir selbst zuzuschreiben. Die Unterstützung durch mein Umfeld war zahlreich, weshalb ich an dieser Stelle einigen Personen meinen aufrichtigen Dank aussprechen möchte.

Allen voran gilt mein Dank meinem Doktorvater Prof. Dr. Manfred Schwaiger. Er gab mir nicht nur die Inspiration für dieses Forschungsvorhaben, sondern stand mir auch stets als Diskussionspartner hilfsbereit zu Seite. Darüber hinaus ermöglichte er mir durch das vertrauensvolle Gewähren von Freiheiten und damit verbundenen Verantwortungen eine reibungslose Anfertigung meiner Dissertation. Gleichzeitig möchte ich meinem Zweitgutachter Prof. Dr. Thomas Hess für die Übernahme des Korreferats und sein Interesse an meiner Arbeit danken.

Des Weiteren möchte ich allen Kolleginnen und Kollegen am Institut meinen Dank aussprechen. Sie hielten mir nicht nur den Rücken frei, sondern boten mir stets ihre Unterstützung und konstruktiven Austausch an. Im Speziellen gilt dies für Matthias Schloderer und Dr. Marko Sarstedt (danke Dir besonders für Motivationsschübe, Ablenkungen, Eingebungen, Geduld und ansteckenden Optimismus). Ebenso danke ich dem gesamten Team der Orientierungsphase für die mentale Unterstützung und Geduld. Frau Gabriela Latinjak gebührt ein besonderer Dank: Als private Lektorin, Ratgeberin, Mentaltrainerin und in vielen weiteren Rollen hat sie einen beträchtlichen Beitrag zu dieser Arbeit geleistet.

Mein größter Dank allerdings gebührt meinen Freunden und meiner Familie. Besonders meinem Vater Dr. Walthari Fuchs danke ich für die unermessliche Unterstützung und den Antrieb, den er mir beibrachte. Meinem Bruder Dr. Maximilian Fuchs danke ich für seine immerwährende und kompromisslose Hilfsbereitschaft und die damit gegebene Kraft.

Ich widme diese Arbeit meiner Mutter Christiane Fuchs (* 1949, † 2006), die deren Fertigstellung leider nicht mehr miterleben durfte. Ich danke Dir für das was ich bin! 


\section{Inhaltsverzeichnis}

Abbildungsverzeichnis .........................................................................

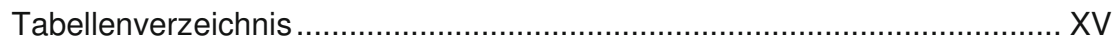

Abkürzungsverzeichnis................................................................... XVII

1 Zielsetzung und Aufbau der Arbeit ............................................... 1

2 Untersuchungsgegenstand.............................................................. 7

2.1 Markenstärke und ihre Messung ................................................ 7

2.1.1 Marke - mehr als nur die Markierung von Produkten ..................... 7

2.1.2 Markenwert und Markenstärke....................................................... 8

2.1.3 Markenbewertungsmodelle .................................................... 11

2.1.4 Messung von Markenstärke …................................................ 19

2.2 Reputation und ihre Messung .................................................... 27

2.2.1 Reputation - mehr als nur das Image eines Unternehmens ........ 27

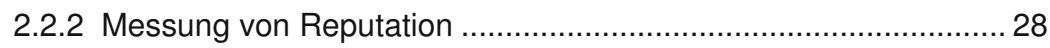

2.3 Differenzierung der Wirkungsbetrachtung nach der

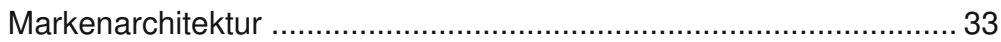

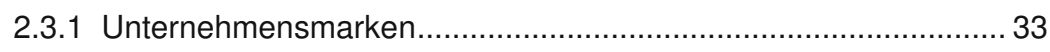

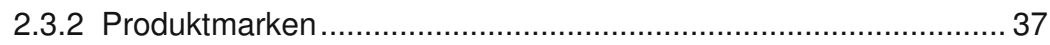

3 Methodische Grundlagen ............................................................. 41

3.1 Modellierung von latenten Konstrukten mit Hilfe von Strukturgleichungsmodellen...................................................... 42

3.1.1 Reflektive versus formative Konstrukte in Strukturgleichungsmodellen 
3.1.2 Varianzbasierte versus kovarianzbasierte Schätzung von Strukturgleichungsmodellen 46

3.1.3 Unbeobachtbare Heterogenitäten in Strukturgleichungsmodellen. 48

3.2 Implizite Messung von Präferenzen mit Conjoint-Analysen 51

3.2.1 Klassische Conjoint-Analyse 52

3.2.1.1 Untersuchungsdesign einer klassischen CA..... .53

3.2.1.2 Analyse von Teilnutzenwerten in der klassischen CA 55

3.2.1.3 Weiterentwicklungen der klassischen CA .57

3.2.2 Choice-based Conjoint-Analyse..... 59

3.2.2.1 Untersuchungsdesign einer CBCA 60

3.2.2.2 Analyse von Teilnutzenwerten in der CBCA 63

3.2.3 Wahl des richtigen Conjoint-Verfahrens 65

3.3 Untersuchung von Ursache-Wirkungs-Beziehungen durch

Experimente 67

3.3.1 Interne und externe Validität bei Experimenten 68

3.3.2 Experimentaldesigns 70

4 Untersuchungsdesign .77

4.1 Reputation und Markenstärke im Strukturgleichungsmodell .77

4.2 Quantifizierung des Außenkriteriums. .79

4.3 Experimenteller Versuchsaufbau zu Produktmarken 81

4.4 Verwendete Stimuli 83

4.4.1 Stimuli zur Analyse der Unternehmensmarken. 84

4.4.2 Stimuli zur Analyse der Produktmarken. 87

4.5 Ziehung der Stichproben und Datenerhebung 89 
5 Der Erklärungsgehalt von Reputation und Markenstärke bei Unternehmensmarken ................................................................... 93

5.1 Zusammensetzung der Stichproben ............................................. 93

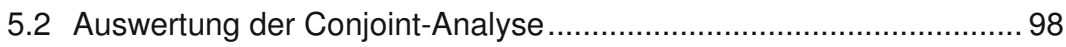

5.3 Betrachtung der Erklärungsanteile im Strukturgleichungsmodell .... 104

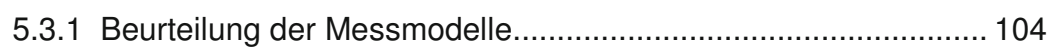

5.3.1.1 Güte der reflektiven Messmodelle........................................ 105

5.3.1.2 Güte der formativen Messmodelle ...................................... 110

5.3.2 Beurteilung der Strukturmodelle.............................................. 113

5.3.2.1 Erklärungsanteil der Reputation......................................... 113

5.3.2.2 Erklärungsanteil der Markenstärke ..................................... 119

5.3.2.3 Gegenüberstellung des Erklärungsgehaltes von Reputation und Markenstärke ............................................................ 121

5.3.3 Analyse unbeobachtbarer Heterogenitäten ............................... 125

5.3.3.1 Aufdeckung von latenten Segmenten ................................. 127

5.3.3.2 Beschreibung der latenten Segmente................................. 131

5.4 Überprüfung der Hypothesen zu Unternehmensmarken ................ 134

\section{Der Transfer zwischen Reputation und Markenstärke bei} Produktmarken ............................................................................. 139

6.1 Zusammensetzung der Stichproben ........................................... 140

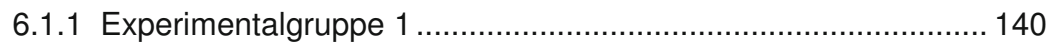

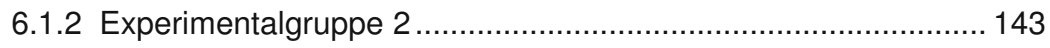

6.2 Überprüfung der Wirksamkeit der Treatments ............................... 144

6.2.1 Beeinflussung der Reputation (EG1) ................................... 145

6.2.2 Beeinflussung der Markenstärke (EG2) ................................ 147 
6.3 Überprüfung der Hypothesen zu Produktmarken 149

6.3.1 Einstellungstransfer von Reputation auf Markenstärke (EG1) .... 150

6.3.1.1 Betrachtung der Gesamtstichprobe (EG1) ........................ 150

6.3.1.2 Vergleiche einzelner Teilstichproben (EG1) ........................ 156

6.3.2 Einstellungstransfer von Markenstärke auf Reputation (EG2) .... 158

6.3.2.1 Betrachtung der Gesamtstichprobe (EG2) ......................... 159

6.3.2.2 Vergleiche einzelner Teilstichproben (EG2) ....................... 162

7 Zusammenfassung und Implikationen............................................. 167

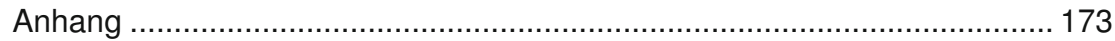

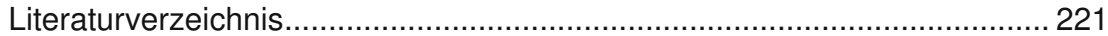




\section{Abbildungsverzeichnis}

Abbildung 1: Studien differenziert nach Markenarchitektur ........................ 4

Abbildung 2: Überblick über den Aufbau der Arbeit ................................... 6

Abbildung 3: Brand Value Chain.......................................................... 18

Abbildung 4: Markenmodell nach AAKER ................................................. 20

Abbildung 5: Markenmodell nach KELLER.............................................. 20

Abbildung 6: Kausalkette des Markeneisberg-Modells.............................. 21

Abbildung 7: Markenmodell nach YoO/DONTHU ....................................... 22

Abbildung 8: Reputationsmodell nach SCHWAIGER................................... 31

Abbildung 9: Überblick über latent class-Verfahren für varianzbasierte

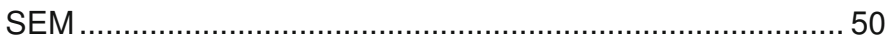

Abbildung 10: Häufige Methoden der Präferenzmessung.......................... 54

Abbildung 11: SOLOMON-Viergruppenversuchsplan .............................. 72

Abbildung 12: Reversed-Treatment-Design .......................................... 73

Abbildung 13: Experimentaldesign zur Wirkungsmessung bei

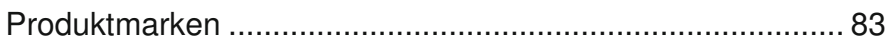

Abbildung 14: Auswahlsituation (Choice-Task) der Choice-based Con-

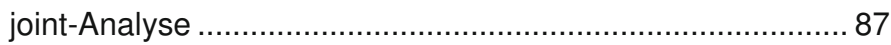

Abbildung 15: Reputationsbeurteilung der bewerteten Unternehmen........... 98

Abbildung 16: Erklärungsanteile der Reputation an product-market outcomes

Abbildung 17: Erklärungsanteile der Markenstärke an product-market outcomes

Abbildung 18: Erklärungsanteile der Reputation und der Markenstärke an product-market outcomes

Abbildung 19: Schrittweise Analyse von unbeobachtbaren Heterogenitäten mit FIMIX-PLS

Abbildung 20: Kombiniertes Modell zur maximalen Varianzaufklärung...... 137 


\section{Tabellenverzeichnis}

Tabelle 1: Maße aus gängigen Markenmessmodellen mit dem Fokus customer mind-set .............................................................. 15

Tabelle 2: Maße aus gängigen Markenmessmodellen mit dem Fokus product-market outcomes ...................................................... 16

Tabelle 3: Maße aus gängigen Markenmessmodellen mit dem Fokus financial market outcomes.................................................... 17

Tabelle 4: Kriterien der Reputationsbewertung Rankings weltweit ............. 28

Tabelle 5: Unterscheidungskriterien zur Konstruktspezifizierung............... 44

Tabelle 6: Übersicht zur Wahl eines geeigneten CA-Verfahrens ................. 66

Tabelle 7: Attributausprägungen der in der Choice-based ConjointAnalyse eingesetzten Stimuli............................................... 86

Tabelle 8: Altersstruktur, Bildungsgrad und berufliche Tätigkeit der Befragten unterteilt nach Branche und Geschlecht ....................95

Tabelle 9: Choice-based Conjoint-Analyse MNL-Schätzung (aggregierte Daten) ................................................................ 100

Tabelle 10: Beurteilung der reflektiven Messmodelle................................... 110

Tabelle 11: Beurteilung der formativen Messmodelle ................................ 112

Tabelle 12: Resultate des Bootstrapping-Verfahrens - total effects der exogenen Treiberkonstrukte der Reputation auf PMO bei gesonderter Betrachtung der Reputation

Tabelle 13: Effektstärken der kompletten Konstrukte Reputation und Markenstärke. 123

Tabelle 14: Resultate des Bootstrapping-Verfahrens - total effects der exogenen Treiberkonstrukte der Reputation auf PMO im Gesamtmodell.

Tabelle 15: Kriterien zur Modellselektion in FIMIX-PLS (Gesamtmodell) ... 129

Tabelle 16: Resultate der FIMIX-PLS Schätzung - Pfadkoeffizienten der Strukturmodelle

Tabelle 17: Deskriptive Auswertung Involvement, Wissen und Erfahrung (EG1)

Tabelle 18: Deskriptive Auswertung Involvement, Wissen und Erfahrung (EG2) 
Tabelle 19: Test der Wirksamkeit der Treatments auf Reputation (EG1) ... 146

Tabelle 20: Test der Wirksamkeit der Treatments auf Markenstärke

(EG2)

Tabelle 21: Test des Transfers von Reputation auf Markenstärke (Gesamtstichprobe EG1).

Tabelle 22: ANOVA zum Vergleich der Markenstärkeveränderungen in den Treatmentgruppen (EG1) 154

Tabelle 23: Korrelation zwischen den Differenzen der Reputations- und der Markenstärkebewertungen aus Pre- und Posttest (EG1) .. 155

Tabelle 24: Überblick über die durchschnittlichen Veränderungen der Bewertungen (EG1) 158

Tabelle 25: Test des Transfers von Markenstärke auf Reputation (Gesamtstichprobe EG2) 159

Tabelle 26: ANOVA zum Vergleich der Reputationsveränderungen in den Treatmentgruppen (EG2) 161

Tabelle 27: Korrelation zwischen den Differenzen der Markenstärke- und der Reputationsbewertungen aus Pre- und Posttest (EG2) ..... 162

Tabelle 28: Überblick über die durchschnittlichen Veränderungen der Bewertungen (EG2) 165

Tabelle 29: Zusammenfassung der Hypothesenprüfung 167 


\section{Abkürzungsverzeichnis}

ACA

Adaptive Conjoint-Analyse

AIC Akaike Information Criterion

AMAC America's Most Admired Companies

Anm. d. Verf. Anmerkung des Verfassers ANOVA Analysis of Variances

BIC Bayes Information Criterion bzw. beiziehungsweise CA Conjoint-Analyse CAIC .Consistent Akaike Information Criterion CBCA Choice-based Conjoint-Analyse $\mathrm{CBC} / \mathrm{HB}$.. Hierarchische Bayes Schätzung in Choice-based Conjoint-Analysen CBSEM covariance-based structural equation model CHAID Chi-squared Automatic Interaction Detector CSR Corporate Social Responsibility DIN . Deutsches Institut für Normung e.V. DEV durchschnittlich erfasste Varianz

EG Experimentalgruppe

ELM Elaboration Likelihood Model

EM expectation-maximisation

EN Entropie-Kriterium et al. und andere etc. et cetera

FIMIX-PLS finite mixture partial least squares GfK Gesellschaft für Konsumforschung GMAC Global Most Admired Companies GoF goodness of fit index HB. Hierarchical Bayes HCA Hybride Conjoint-Analyse HILCA Hierarchische Individualisierte Limit Conjoint-Analyse IAS International Accounting Standards IBR individual behaviour rule i. V. m. in Verbindung mit 

Limit Conjoint-Analyse multiple indicators multiple causes $\mathrm{MNL}$ multinomial logit non-governmental organization OLS .ordinary least squares

o. V. ohne Verfasser

PLS partial least squares

PMO product-market outcomes

SEM .structural equation model SIBR set of individual behaviour rules sog. so genannt(-e/-er)

u. a. unter anderem usw. und so weiter u. v. m. und viele mehr v. a. .vor allem vgl. vergleiche VIF Varianzinflationsfaktor Vol. Volume z.B. zum Beispiel 